To appear in the International Journal of Systems Science

Vol. 00, No. 00, Month 20XX, 1-19

\title{
Towards Probabilistic Synchronization of Local Controllers
}

\author{
Randa Herzallah ${ }^{\mathrm{a} *}$ and Miroslav Kárnýb \\ ${ }^{a}$ NCRG, Aston University, Birmingham, UK; ${ }^{b}$ Institute of Information Theory and Automation, Prague, \\ $C Z$
}

(Received 00 Month 20XX; final version received 00 Month 20XX)

\begin{abstract}
The traditional use of global and centralised control methods, fails for large, complex, noisy and highly connected systems, which typify many real world industrial and commercial systems. This paper provides an efficient bottom up design of distributed control in which many simple components communicate and cooperate to achieve a joint system goal. Each component acts individually so as to maximise personal utility whilst obtaining probabilistic information on the global system merely through local message-passing. This leads to an implied scalable and collective control strategy for complex dynamical systems, without the problems of global centralised control. Robustness is addressed by employing a fully probabilistic design, which can cope with inherent uncertainties, can be implemented adaptively and opens a systematic rich way to information sharing. This paper opens the foreseen direction and inspects the proposed design on a linearised version of coupled map lattice with spatiotemporal chaos. A version close to linear quadratic design gives an initial insight into possible behaviours of such networks.
\end{abstract}

\section{Introduction}

Many systems in nature, technology and society can be regarded as complex networks which consist of a large number of elements interacting with each other. The scale and nature of such systems make them pervasive, inherently nonlinear, coupled and operating under high levels of uncertainty. These attributes make the analysis, estimation and especially control of such systems a significant challenge which has yet to be adequately addressed. Several estimation and control methods have been proposed in the literature, including synchronising chaos Boccaletti et al. (2002); Tan et al. (2003), pinning control Porfiri \& diBernardo (2008), multi-agent control Broek et al. (2008), probabilistic control Herzallah (2011); Herzallah \& Lowe (2003, 2007), decentralised control Šiljak \& Zečević (2005), and distributed control Wang et al. (2014). However, these control techniques suffer from either representing single-agent architectures as far as the controller design is concerned, which are centralised and so complete observation of the global state must be known, or are decentralised and decisions are based only on disconnected knowledge.

This paper proposes a third alternative which: a) has not been explored before, and b) provides potentially new insight that could lead to significant advances in current control theory. No centralised control is assumed and overall behaviour emerges from the low level component interaction. In particular, we propose decomposing the global control problem by designing multicontrollers at the low component levels that act to maximise personal utility whilst obtaining probabilistic information on the global system through local message-passing. This leads to an implied collective control strategy without the problems of global centralised control. This idea of combining distributed control and probabilistic message-passing allows the inclusion of multiple and diverse noise sources, asynchronous information update, and non-linear and non-stationary dynamics; thereby circumvents many of the problems of current control theory.

*Corresponding author. Email: r.herzallah@aston.ac.uk 
Access to cheap computational and communication resources now allows improvement of the overall behaviour of the network of such controlled architectures by allowing the individual nodes to share information with their neighbours without aiming for unattainable global solutions. The synchronisation can then be achieved via message-passing. It is one of the new classes of distributed methods for managing systems with large ensembles of interconnected nodes Aji \& McEliece (2000) where information is retrieved and disseminated in a consistent probabilistic fashion. This approach has emerged independently in a number of fields, including: communications theory Gallager (1963), artificial intelligence Pearl (1988), and statistical physics Parisi et al. (1987). However, the techniques and their potential generalisations have not yet penetrated into the control community.

An objective of this paper is to generalise these methods in the context of distributed probabilistic control by extending them from a passive to an active domain. This approach of a collection of distributed probabilistic controllers passing information through probabilistic "messages", can decompose the control of the large-scale networks into a collection of smaller control problems, one for each connected subnetwork in the system. These sub-control problems are treated independently and can therefore be analysed and implemented individually.

This paper therefore proposes a novel way of reducing the amount of computation required in the optimisation, control, and estimation of complex networked systems. Instead of designing a global probabilistic controller as in the fully probabilistic design (FPD) of single dynamical systems, we propose designing distributed multicontrollers in such a way that the global behaviour of the complex system is achieved. Our assumption is based on targeting the global performance of complex systems through local actions designed to control the subsystems constituting the complex system. According to this assumption, the complex property (global behavioural performance) can be realised by understanding simpler subsystems behaviour (the localised behaviour of controlled subsystems). Thus, to realise our hypothesis we aim to design and develop distributed control methods for the individual subsystems so that the desired global behavioural performance of the system is achieved. Nodes exchange information or messages with their neighbours according to the topology of the graph. The messages are then updated iteratively in a probabilistic framework, and are used to estimate the dynamics of decision variables in the network. Fully probabilistic design of controllers (FPD) Herzallah \& Kárný (2011); Kárńy (1996) is adopted here for designing each controller. These controllers are then equipped with probabilistic message passing techniques in order to exchange information about the various subsystems constituting the complex dynamical system. In FPD, the optimal controller is the minimiser of the Kulback-Leibler divergence of the probability density function describing closed loop dynamics of the controlled system to its ideal counterpart. Its relevance is due to: i) its ability to cope with stochastic nature of the controlled nodes, ii) the direct way to adaptive controllers, iii) use of the unified probabilistic language for modelling and control-aims expression, hence, significantly simplifying the use message-passing schemes.

The final aim of the reported research is quite ambitious. This paper provides a basic understanding of the proposed distributed probabilistic control formulation and message passing. For that purpose, the foreseen design is inspected on a simple linear quadratic example. The proposed distributed probabilistic control methodology is compared with the standard pinning control technique. Simulation results indicate the efficacy of the distributed controllers in achieving the optimal performance of the controlled system with potentially less computational and design efforts when compared to pinning control.

Preliminaries are given in Section 2. Section 3 formulates and solves the problem. Section 4 recalls classical linear quadratic design in non-classical fully probabilistic formulation. Also, both centralised and distributed versions are presented and discussed. Section 5 provides the algorithm of the FPD distributed control method. Coupled map lattice network and numerical experiments are given in Section 6. Section 7 provides concluding remarks. 


\section{Preliminaries}

In this paper we consider the general form of stochastic dynamical system which consists of large number of states, $x_{t} \in \mathbb{R}^{n}$ that are determined by the system inputs $u_{t} \in \mathbb{R}^{m}$. The system exhibits random behaviour, therefore, only the conditional probability density function (pdf), of the future state $x_{t+1}$ conditioned on the current input $u_{t}$ and the current state $x_{t}$ can be specified at each instant of time $t \in\{1, \ldots, H\}$, where $H$ is a finite control horizon,

$$
s\left(x_{t+1} \mid u_{t}, x_{t}\right)
$$

The objective is to design a randomised controller, described by the conditional pdf,

$$
c\left(u_{t} \mid x_{t}\right), \quad t=1, \ldots, H
$$

that shapes the joint pdf of the closed loop behaviour of the dynamical system. The optimal controller should make this joint pdf as close as possible to a desired pdf. To achieve this control aim the FPD approach considers the minimisation of the Kullback-Leibler divergence (KLD) between the actual joint pdf $f(D)$ of the observed data, $D=(x(1), \ldots, x(H), u(1), \ldots, u(H))$ and a predefined ideal (desired) pdf ${ }^{I} f(D)$,

$$
\mathcal{D}\left(f \|{ }^{I} f\right) \equiv \sum_{t=1}^{H} \int f(D) \ln \left(\frac{f(D)}{I f(D)}\right) \mathrm{d} D .
$$

This design methodology was originally presented in Kárńy (1996), where the optimal probabilistic controller is derived. The joint pdf $f(D)$ of the data sequence $D$, is the most complete probabilistic description of the closed loop system behavior. By using the chain rule Peterka $(1981), f(D)$ can be factorised as follows,

$$
f(D)=s\left(x_{t+1} \mid u_{t}, x_{t}\right) c\left(u_{t} \mid x_{t}\right) .
$$

The factors $s\left(x_{t+1} \mid u_{t}, x_{t}\right)$ defined in (1), and $c\left(u_{t} \mid x_{t}\right)$ defined in (2) describe the pdfs of the system dynamics and the controller respectively as stated above. Similarly the ideal joint pdf, ${ }^{I} f(D)$ can be factorised as follows,

$$
{ }^{I} f(D)={ }^{I} s\left(x_{t+1} \mid u_{t}, x_{t}\right){ }^{I} c\left(u_{t} \mid x_{t}\right),
$$

where ${ }^{I} s\left(x_{t+1} \mid u_{t}, x_{t}\right)$ and ${ }^{I} c\left(u_{t} \mid x_{t}\right)$ represent the pdfs of the desired system dynamics and ideal controller, respectively.

Minimisation of (3) with respect to randomised control input (2) can be obtained recursively from the following backward recurrence equation, Herzallah \& Kárný (2011),

$$
\begin{aligned}
& -\ln \left(\gamma\left(x_{t}\right)\right)=\min _{c\left(u_{t} \mid x_{t}\right)} \int s\left(x_{t+1} \mid u_{t}, x_{t}\right) c\left(u_{t} \mid x_{t}\right) \times \underbrace{[\underbrace{\ln \left(\frac{s\left(x_{t+1} \mid u_{t}, x_{t}\right) c\left(u_{t} \mid x_{t}\right)}{I_{s}\left(x_{t+1} \mid u_{t}, x_{t}\right) I^{I} c\left(u_{t} \mid x_{t}\right)}\right)}_{\text {optimal cost-to-go }}}_{\equiv \text { partial } \operatorname{cost} U\left(x_{t+1}, u_{t}, x_{t}\right)} \\
& -\underbrace{\ln \left(\gamma\left(x_{t+1}\right)\right)}] \mathrm{d}\left(x_{t+1}, u_{t}\right) .
\end{aligned}
$$


where we have substituted (4) and (5) in (3), and where

$$
-\ln \left(\gamma\left(x_{t}\right)\right)=\min _{c\left(u_{\tau} \mid x_{\tau}\right)_{\tau \geq t}^{H}} \sum_{\tau=t}^{H} \int \mathcal{D}\left(f \|{ }^{I} f\right)
$$

is the expected minimum cost-to-go function for arbitrary $\tau \in\{1, \ldots, H\}$. Full derivation of (6) can be found in Herzallah \& Kárný (2011).

The pdf of optimal controller, $c^{*}\left(u_{t} \mid x_{t}\right)$, minimizing the cost-to-go function (6) is determined by the following backward recursion, see e.g. Herzallah \& Kárný (2011); Herzallah (2013) ${ }^{1}$,

$$
\begin{aligned}
c^{*}\left(u_{t} \mid x_{t}\right) & =\frac{I_{c}\left(u_{t} \mid x_{t}\right) \exp \left[-\beta\left(u_{t}, x_{t}\right)\right]}{\gamma\left(x_{t}\right)}, \\
\gamma\left(x_{t}\right) & =\int{ }^{I} c\left(u_{t} \mid x_{t}\right) \exp \left[-\beta\left(u_{t}, x_{t}\right)\right] \mathrm{d} u_{t}, \\
\beta\left(u_{t}, x_{t}\right) & =\int s\left(x_{t+1} \mid u_{t}, x_{t}\right)\left[\ln \left(\frac{s\left(x_{t+1} \mid u_{t}, x_{t}\right)}{I_{s}\left(x_{t+1} \mid u_{t}, x_{t}\right)}\right)-\ln \left(\gamma\left(x_{t+1}\right)\right)\right] \mathrm{d} x_{t+1} .
\end{aligned}
$$

In large scale systems, the above methodology for designing probabilistic controllers becomes formidable. This is typically because of the large number of nodes where it becomes impossible to add controllers to all nodes. Therefore, the FPD has been extended in Herzallah (2012) to control complex stochastic dynamical networks via pinning control. Optimized probabilistic pinning control laws have the same form as specified by equation (7), but inputs are applied only to a fraction of nodes in the network. Although pinning control requires a significantly smaller number of controllers, it requires that the network need not be controllable with a technically feasible amount of controlled nodes.

Thus in this paper, we propose an alternative formulation that minimises the computational and design efforts in large scale systems via distributed control architecture. The proposed distributed control method will be discussed in the next section. It will be developed using the concept of FPD.

\section{Problem Formulation and Solution}

Within the proposed distributed control framework, we consider that the complex system is decomposed into $K$ subsystems, each controlled independently by a probabilistic controller. The individual probabilistic controllers consider the dynamics of their subpart of the system only and treat the dynamics of other subsystems as a measurable external disturbance. These subsystems share information with each other to achieve a global system goal in a cooperative manner where various subsystems have access to different and local information. The message passing method to be employed in this paper includes subsystems that constitute the complex system as individual nodes in order to optimise and manage the global system goal through the local control of subsystems.

In the following discussion the subscript $(k)$ refers to the $k$ th subsystem of the complex system, whereas the subscript $k$ refers to the $k$ th subpart of the overall system state. To further clarify the notation, the architecture of this framework for a two node system is shown in Figure 1. Following this notation, the $k$ th subsystem is to be controlled by a randomised controller described by pdf $c_{(k)}\left(u_{t ;(k)} \mid x_{t}\right), k \in\{1, \ldots, K\}$ with possibly large $K$. The overall input $u_{t}$ is composed of nonoverlapping inputs generated by respective controllers $u_{t}^{T}=\left(u_{t ;(1)}^{T}, \ldots, u_{t ;(K)}^{T}\right)$ where $T$ denotes

${ }^{1}$ Theorem 1 below indicates how to get this recursion. The general case with noisy measurements of the state is in Kárný \& Guy (2006). 
transposition. The $k$ th controller is designed to control the $k$ th subpart of the overall state $x_{t+1 ; k}$, i.e. $x_{t+1}^{T}=\left(x_{t+1 ; 1}^{T}, \ldots, x_{t+1 ; K}^{T}\right)$. It models the rest of the state as a measurable external disturbance. This means that the $k$ th controller describes the subsystem state by the pdf $s_{(k)}\left(x_{t+1} \mid u_{t ;(k)}, x_{t}\right)$ structured as follows,

$$
s_{(k)}\left(x_{t+1} \mid u_{t ;(k)}, x_{t}\right)=s_{(k)}\left(x_{t+1 ; k} \mid u_{t ;(k)}, x_{t}\right) s_{(k)}\left(x_{t+1 ;-k} \mid x_{t ;-k}\right),
$$

where $x_{t+1 ;-k}$ is the state part obtained by removing $x_{t+1 ; k}$ from the overall state $x_{t+1}$. To achieve the objective of considering $x_{t+1 ;-k}$ as a measurable external disturbance, the ideal description of pdf of the system dynamics is defined as follows,

$$
{ }^{I} s_{(k)}\left(x_{t+1} \mid u_{t ;(k)}, x_{t}\right)={ }^{I} s_{(k)}\left(x_{t+1 ; k} \mid u_{t ;(k)}, x_{t}\right){ }^{I} s_{(k)}\left(x_{t+1 ;-k} \mid x_{t ;-k}\right) .
$$

This definition of the ideal pdf of the system dynamics means that the pdf describing the "external" variables $x_{t+1 ;-k}$ is taken to be the same as their ideal (desired) pdf Kárný et al. (2006), thus not influencing their behavioral outputs.

Following on from this formulation, the FPD leading to the $k$ th controller is described by the following theorem.

Theorem 1 If the $k$ th subsystem: a) selects inputs $u_{t ;(k)}$, b) models the state evolution by (8), c) expresses its aims by the ideal system model (9) and by an ideal controller ${ }^{I} c_{(k)}\left(u_{t ;(k)} \mid x_{t}\right)$, then the optimal controller minimizing the cost-to-go function (6) is given by,

$$
\begin{gathered}
c_{(k)}^{*}\left(u_{t ;(k)} \mid x_{t}\right)=\frac{{ }^{I} c_{(k)}\left(u_{t ;(k)} \mid x_{t}\right) \exp \left[-\beta\left(u_{t ;(k)}, x_{t}\right)\right]}{\gamma_{(k)}\left(x_{t}\right)} \\
\gamma_{(k)}\left(x_{t}\right)=\int{ }^{I} c_{(k)}\left(u_{t ;(k)} \mid x_{t}\right) \exp \left[-\beta\left(u_{t ;(k)}, x_{t}\right)\right] \mathrm{d} u_{t ;(k)} \\
\beta\left(u_{t ;(k)}, x_{t}\right)=\int s_{(k)}\left(x_{t+1 ; k} \mid u_{t ;(k)}, x_{t}\right)\left[\ln \left(\frac{s_{(k)}\left(x_{t+1 ; k} \mid u_{t ;(k)}, x_{t}\right)}{I_{s(k)}\left(x_{t+1 ; k} \mid u_{t ;(k)}, x_{t}\right)}\right)-\ln \left(\tilde{\gamma}_{(k)}\left(x_{t+1 ; k}, x_{t ;-k}\right)\right)\right] \mathrm{d} x_{t+1 ; k} \\
\ln \left(\tilde{\gamma}_{(k)}\left(x_{t+1 ; k}, x_{t ;-k}\right)\right)=\int s_{(k)}\left(x_{t+1 ;-k} \mid x_{t ; k}\right) \ln \left(\gamma_{(k)}\left(x_{t+1}\right)\right) \mathrm{d} x_{t+1 ;-k} .
\end{gathered}
$$

Proof: The result is implied by the following sequence of equalities in which one cost-to-go function (6) is dedicated to obtain the optimal control solution for each of the $k$ th controllers. We also use Fubini's theorem on multiple integration Rao (1987), marginalisation, normalisation and the chain rule of pdfs Peterka (1981) together with conditional independence expressed by the assumption stated above.

Hence the $k$ th cost-to-go function for the assumed models of the $k$ th subpart of the system can be obtained from (6) to yield,

$$
\begin{gathered}
\ln \left(\gamma_{(k)}\left(x_{t}\right)\right)=\min _{c_{(k)}\left(u_{t ;(k)} \mid x_{t}\right)} \int s_{(k)}\left(x_{t+1} \mid u_{t ;(k)}, x_{t}\right) c_{(k)}\left(u_{t ;(k)} \mid x_{t}\right) \\
\times[\underbrace{\ln \left(\frac{s_{(k)}\left(x_{t+1} \mid u_{t ;(k)}, x_{t}\right) c_{(k)}\left(u_{t ;(k)} \mid x_{t}\right)}{I_{s_{(k)}}\left(x_{t+1} \mid u_{t ;(k)}, x_{t}\right)^{I} c_{(k)}\left(u_{t ;(k)} \mid x_{t}\right)}\right)}_{\equiv \text { partial cost } U\left(x_{t+1}, u_{t ;(k)}, x_{t}\right)}-\underbrace{\ln \left(\gamma_{(k)}\left(x_{t+1}\right)\right)}_{\text {optimal cost-to-go }}] \mathrm{d}\left(x_{t+1}, u_{t ;(k)}\right) .
\end{gathered}
$$

Using the $k$ th state model (8), the ideal distribution of the $k$ th subsystem (9), the ideal distribution 
of the $k$ th controller ${ }^{I} c_{(k)}\left(u_{t ;(k)} \mid x_{t}\right)$, and the chain rule, equation (11) can be rewritten as,

$$
\begin{aligned}
& -\ln \left(\gamma_{(k)}\left(x_{t}\right)\right)=\int s_{(k)}\left(x_{t+1 ; k} \mid u_{t ;(k)}, x_{t}\right) s_{(k)}\left(x_{t+1 ;-k} \mid x_{t}\right) c_{(k)}\left(u_{t ;(k)} \mid x_{t}\right) \times\left[\ln \left(\frac{s_{(k)}\left(x_{t+1 ; k} \mid u_{t ;(k)}, x_{t}\right)}{I_{s_{(k)}}\left(x_{t+1 ; k} \mid u_{t ;(k)}, x_{t}\right)}\right)\right. \\
& \left.+\ln \left(\frac{c_{(k)}\left(u_{t ;(k)} \mid x_{t}\right)}{I_{(k)}\left(u_{t ;(k)} \mid x_{t}\right)}\right)-\ln \left(\gamma_{(k)}\left(x_{t+1}\right)\right)\right] \mathrm{d}\left(x_{t+1 ; k}, x_{t+1 ;-k}, u_{t ;(k)}\right) .
\end{aligned}
$$

Now using Fubini's theorem, we introduce the following definitions

$$
\begin{gathered}
\ln \left(\tilde{\gamma}_{(k)}\left(x_{t+1 ; k}, x_{t ;-k}\right)\right)=\int s_{(k)}\left(x_{t+1 ;-k} \mid x_{t ;-k}\right) \ln \left(\gamma_{(k)}\left(x_{t+1}\right)\right) \mathrm{d} x_{t+1 ;-k} \\
\beta\left(u_{t ;(k)}, x_{t}\right)=\int s_{(k)}\left(x_{t+1 ; k} \mid u_{t ;(k)}, x_{t}\right)\left[\begin{array}{c}
\left.\ln \left(\frac{s_{(k)}\left(x_{t+1 ; k} \mid u_{t ;(k)}, x_{t}\right)}{I_{(k)}\left(x_{t+1 ; k} \mid u_{t ;(k)}, x_{t}\right)}\right)-\ln \left(\tilde{\gamma}_{(k)}\left(x_{t+1 ; k}, x_{t ;-k}\right)\right)\right] \mathrm{d} x_{t+1 ; k} \\
s_{(k)}\left(x_{t+1 ; k} \mid u_{t ;(k)}, x_{t}\right)
\end{array}\right. \\
=\int s_{(k)}\left(x_{t+1 ; k} \mid u_{t ;(k)}, x_{t}\right) \ln \left(\frac{\tilde{r}_{(k)}\left(x_{t+1 ; k} \mid u_{t ;(k)}, x_{t}\right) \tilde{\gamma}_{(k)}\left(x_{t+1 ; k}, x_{t ;-k}\right)}{I_{s_{(k+1 ; k}}}\right) \mathrm{d} x_{t+1}
\end{gathered}
$$

Substitution of (14) and (13) in (12) gives,

$$
\begin{aligned}
& -\ln \left(\gamma_{(k)}\left(x_{t}\right)\right)=\int c_{(k)}\left(u_{t ;(k)} \mid x_{t}\right)\left[\beta\left(u_{t ;(k)}, x_{t}\right)+\ln \left(\frac{c_{(k)}\left(u_{t ;(k)} \mid x_{t}\right)}{I_{(k)}\left(u_{t ;(k)} \mid x_{t}\right)}\right)\right] \mathrm{d} u_{t ;(k)} \\
& =\int c_{(k)}\left(u_{t ;(k)} \mid x_{t}\right) \ln \left(\frac{c_{(k)}\left(u_{t ;(k)} \mid x_{t}\right)}{I_{(k)}\left(u_{t ;(k)} \mid x_{t}\right) \exp \left(-\beta\left(u_{t ;(k)}, x_{t}\right)\right)}\right) \mathrm{d} u_{t ;(k)}
\end{aligned}
$$

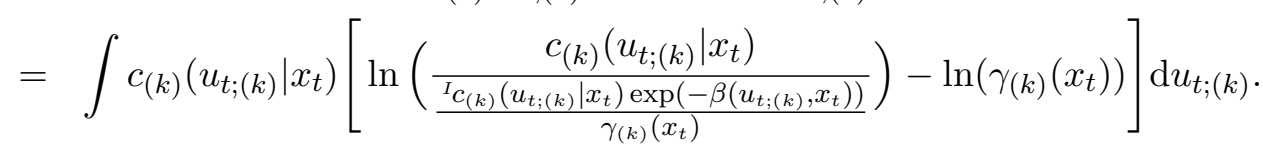

By adding and subtracting $\ln \left(\gamma_{(k)}\left(x_{t}\right)\right)$, the first term in (15) has become a conditional version of the KL divergence. The independence of $\ln \left(\gamma_{(k)}\left(x_{t}\right)\right)$ on the optimised $c_{(k)}\left(u_{t ;(k)} \mid x_{t}\right)$ implies that the expression is minimised by the claimed pdf (10).

Remark 1: The occurrence of the modified cost-to-go $\ln \tilde{\gamma}_{(k)}\left(x_{t+1 ; k}, x_{t ;-k}\right)$ is the key modification over the standard FPD induced by treating $x_{t+1 ;-k}$ as an external variables. The model of the external variables $s_{(k)}\left(x_{t+1 ;-k} \mid x_{t ;-k}\right)$ maps $\ln \gamma_{(k)}$ to $\ln \tilde{\gamma}_{(k)}$ by averaging over the external variables. It substantially decreases the computational load as shown on the FPD version leading to the standard linear Gaussian quadratic design, see Section 4.

Remark 2: To exploit the explorative property of the FPD, control inputs can be generated by sampling from the estimated conditional density, $c_{(k)}^{*}\left(u_{t ;(k)} \mid x_{t}\right)$ of the optimal controller. Depending on the nature of the control problem, more specific quantities can also be calculated. One of the simplest of these is the mean corresponding to the conditional average of control inputs. 


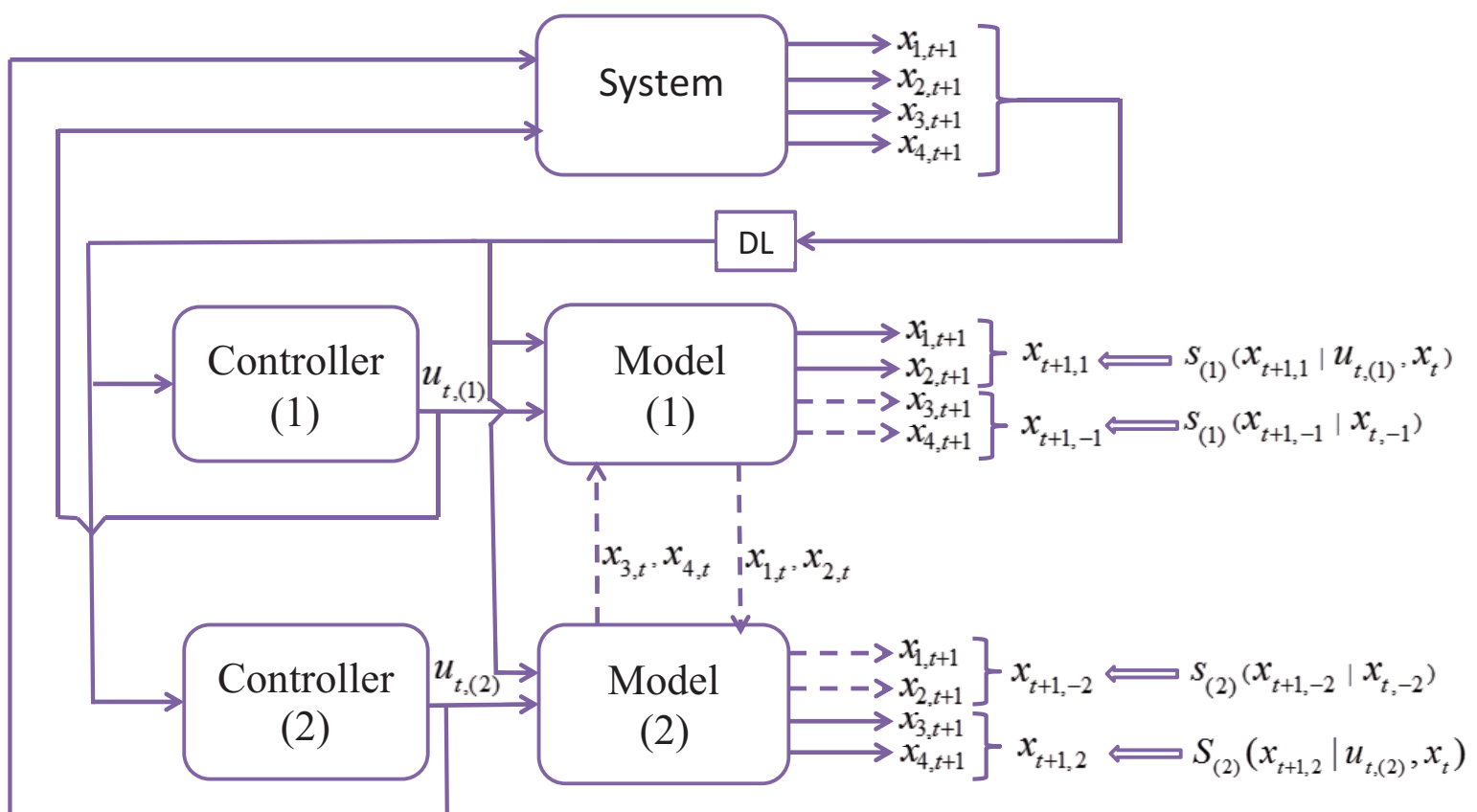

Figure 1. The architecture of the proposed distributed control for a two node system. The solid lines at the outputs of the two models are the estimation of the $k$ th subpart of the system, while the dashed lines at the output of the two models are the estimation of the external variables by these models, obtained by message-passing. Dashed lines between the two models are the communicated messages between them.

\section{Linear Gaussian Quadratic Design}

To demonstrate the distributed control development discussed in the previous section, we consider the regulation problem of a stochastic linear Gaussian state space model described by,

$$
\begin{aligned}
x_{t+1} & =A x_{t}+B u_{t}+\omega_{t+1}, \\
s\left(x_{t+1} \mid x_{t}, u_{t}\right) & \rightsquigarrow \mathcal{N}_{x_{t+1}}\left(A x_{t}+B u_{t}, \Sigma\right),
\end{aligned}
$$

where $A$ and $B$ are the state and control matrices respectively, $\omega_{t+1}$ is zero mean white normal innovations, and $\Sigma$ is the covariance of the innovations. The matrices $A, B$, and $\Sigma>0$ are assumed to be known.

For the considered regulation problem, the system is initially in state $x_{t}$ and the aim is to return the system state to the origin. Before we discuss the distributed control solution for this regulation problem we briefly review the standard solution to the problem using the global FPD control method.

\subsection{Global FPD Control Method}

The standard solution to the regulation problem described above using global FPD control method starts by specifying the ideal distribution of the system states given in equation (16). This ideal 
state distribution is assumed to be given by

$$
{ }^{I} s\left(x_{t+1} \mid u_{t}, x_{t}\right)=\mathcal{N}_{x_{t+1}}(0, \Sigma) \text {. }
$$

It reflects the regulation problem with the realistic aim of reaching the zero state, with a spread being determined by the covariance of the innovations, $\Sigma$.

The randomized controller to be designed is described by,

$$
\begin{aligned}
c\left(u_{t} \mid x_{t}\right) & \rightsquigarrow \mathcal{N}_{u_{t}}\left(C x_{t}, \Gamma\right) \\
u_{t} & =C x_{t}+\epsilon_{t},
\end{aligned}
$$

where $C$ is the matrix of the controller parameters, $\epsilon_{t}$ is zero mean white normal innovations, and $\Gamma$ is the covariance of the innovations of control. The distribution of the ideal controller is assumed to be,

$$
{ }^{I} c\left(u_{t} \mid x_{t}\right)=\mathcal{N}_{u_{t}}\left(0,{ }^{I} \Gamma\right)
$$

where ${ }^{I} \Gamma$ specifies the allowed range of optimal inputs. By using the randomized controller (18), the stochastic equation of the system state (16) can be recast as follows:

$$
x_{t+1}=(A+B C) x_{t}+B \epsilon_{t}+\omega_{t+1} .
$$

If the pair $(A, B)$ is stabilisable, i.e. if there is such a controller $C$ that makes all eigenvalues of the matrix $A+B C$ lie inside the unit circle then the optimal randomised controller as can be obtained from the evaluation of (7) for horizon $H \rightarrow \infty$ is stabilising and has the form Herzallah (2012),

$$
u_{t}=C x_{t}+\epsilon_{t},
$$

with

$$
\begin{aligned}
C & =-\left(B^{T} M B+B^{T} \Sigma^{-1} B+{ }^{I} \Gamma^{-1}\right)^{-1}\left(B^{T} M A+B^{T} \Sigma^{-1} A\right), \\
M & =A^{T} \Sigma^{-1} A+A^{T} M A-\left(A^{T} M B+A^{T} \Sigma^{-1} B\right)\left(B^{T} M B+B^{T} \Sigma^{-1} B+{ }^{I} \Gamma^{-1}\right)^{-1} \\
& \times\left(B^{T} M A+B^{T} \Sigma^{-1} A\right), \\
\Gamma & =\left(B^{T} M B+B^{T} \Sigma^{-1} B+{ }^{I} \Gamma^{-1}\right)^{-1}
\end{aligned}
$$

where $M$ is the matrix of the attained quadratic cost function,

$$
-\ln \left(\gamma\left(\mathbf{x}_{t}\right)\right)=0.5 \mathbf{x}_{t}^{T} M \mathbf{x}_{t}+Q_{0}
$$

and where $Q_{0} \geq 0$ is some positive constant. The derived solution (23) coincides with the stationary algebraic Riccati equation for linear quadratic design Meditch (1969).

\subsection{Distributed FPD Control Method}

The application of FPD distributed controllers to regulate the system (16) is carried out by designing $K$ randomised controllers described by,

$$
\begin{aligned}
c_{(k)}\left(u_{t ;(k)} \mid x_{t}\right) & \rightsquigarrow \mathcal{N}_{u_{t ;(k)}}\left(C_{(k)} x_{t}, \Gamma_{(k)}\right) \\
u_{t ;(k)} & =C_{(k)} x_{t}+\epsilon_{t ;(k)},
\end{aligned}
$$


where $k \in\{1, \ldots, K\}, C_{(k)}$ is the matrix of the $k$ th controller parameters, $\epsilon_{t ;(k)}$ is zero mean white normal innovations of the $k$ th control input, and $\Gamma_{(k)}$ is the covariance of the innovation of $k$ th control. Each of these randomized controllers is responsible for controlling the state values of the corresponding $k$ th subsystem while not influencing the rest of the states $x_{t+1 ;-k}$. As such, $K$ models to estimate the dynamics of the $K$ subsystems must be identified, where one model is to be identified for each randomized controller. The $k$ th model of the $k$ th subsystem describes the small controlled subpart $x_{t+1 ; k}$ of the state, while models the rest of the state $x_{t+1 ;-k}$ as an external observable disturbance,

$$
\begin{aligned}
x_{t+1 ;(k)} & =A_{(k)} x_{t}+B_{(k)} u_{t ;(k)}+\omega_{t+1 ;(k)} \\
s_{(k)}\left(x_{t+1} \mid u_{t ;(k)}, x_{t}\right) & =s_{(k)}\left(x_{t+1 ; k} \mid u_{t ;(k)}, x_{t}\right) s_{(k)}\left(x_{t+1 ;-k} \mid x_{t ;-k}\right) \\
& \rightsquigarrow \mathcal{N}_{x_{t+1 ; k}}\left(A_{(k) ; k} x_{t}+B_{(k) ; k} u_{t ;(k)}, \Sigma_{(k) ; k}\right) \mathcal{N}_{x_{t+1 ;-k}}\left(A_{(k) ;-k} x_{t ;-k}, \Sigma_{(k) ;-k}\right),
\end{aligned}
$$

where $A_{(k) ; k}$ is the state matrix from the $k$ th model related to the $k$ th (controlled) subpart of the state, $A_{(k) ;-k}$ is the state matrix from the $k$ th model related to the external variables of the state, $B_{(k) ; k}$ is the control matrix from the $k$ th model related to the controlled states, $\Sigma_{(k) ; k}$ is the covariance matrix from the $k$ th model related to the controlled states, and $\Sigma_{(k) ;-k}$ is the covariance matrix from the $k$ th model related to the external variables. Following the discussion in Section 3 the ideal distribution of the closed loop model of the $k$ th subsystem does not consider the synchronisation of the "external" variables $x_{t+1 ;-k}$, i.e. the pdf modelling the external variables is taken to be equal to the ideal (desired) pdf of external variables as follows,

$$
\begin{aligned}
& I_{s_{(k)}}\left(x_{t+1 ; k} \mid u_{t ;(k)}, x_{t}\right) s_{(k)}\left(x_{t+1 ;-k} \mid x_{t ;-k}\right) \\
& \mathcal{N}_{x_{t+1 ; k}}\left(0, \Sigma_{(k) ; k}\right) \mathcal{N}_{x_{t+1 ;-k}}\left(A_{(k) ;-k} x_{t ;-k}, \Sigma_{(k) ;-k}\right) .
\end{aligned}
$$

It reflects the aim of regulating $x_{t+1 ; k}$ to zero while treating $x_{t+1 ;-k}$ as an external variable. The distribution of the $k$ th ideal controller is assumed to be,

$$
{ }^{I} c_{(k)}\left(u_{t ;(k)} \mid x_{t}\right)=\mathcal{N}_{u_{t ;(k)}}\left(0,{ }^{I} \Gamma_{(k)}\right) .
$$

By associating the $k$ th controller (25) with its $k$ th model (26), the stochastic equation (26) of the $k$ th model can be recast as follows,

$$
x_{t+1 ;(k)}=\left(A_{(k)}+B_{(k)} C_{(k)}\right) x_{t}+B_{(k)} \epsilon_{t ;(k)}+\omega_{t ;(k)} .
$$

Similarly, under the assumption that All eigenvalues of the matrix $A_{(k)}+B_{(k)} C_{(k)}$ in (29) lie inside the unit circle, a stabilisable randomised control $c_{(k)}\left(u_{t ;(k)} \mid x_{t}\right)$ can be obtained from (10). This is achieved by substituting the assumed model of the subpart of the system states (26), the ideal distribution of the $k$ th subsystem (27), and the ideal distribution of the $k$ th controller (28) in (10). Hence, the optimal feedback control law can be specified in the following theorem.

Theorem 2 For the distributed type linear Gaussian FPD controller with external variables considered in this paper, if the $k$ th node: a) selects inputs $u_{t ;(k)}$, b) models the state evolution by (26), c) expresses its aims by the ideal system model $(27)$ and by an ideal controller ${ }^{I} c_{(k)}\left(u_{t ;(k)} \mid x_{t}\right)$, then the optimal controller minimizing the cost-to-go function (11) is given by either one of the two equivalent forms,

I. Form 1 of the optimal controller

$$
u_{t ;(k)}=C_{(k)} x_{t}+\epsilon_{t ;(k)}
$$


with

$$
\begin{aligned}
C_{(k)} & =-\left(B_{(k)}^{T} M_{(k)} B_{(k)}+B_{(k)}^{T} \Sigma_{k}^{-1} B_{(k)}+{ }^{I} \Gamma_{(k)}^{-1}\right)^{-1}\left(B_{(k)}^{T} M_{(k)} A_{(k)}+B_{(k)}^{T} \Sigma_{k}^{-1} A_{(k)}\right), \\
M_{(k)} & =A_{(k)}^{T} \Sigma_{k}^{-1} A_{(k)}+A_{(k)}^{T} M_{(k)} A_{(k)}-\left(A_{(k)}^{T} M_{(k)} B_{(k)}+A_{(k)}^{T} \Sigma_{k}^{-1} B_{(k)}\right) \\
& \times\left(B_{(k)}^{T} M_{(k)} B_{(k)}+B_{(k)}^{T} \Sigma_{k}^{-1} B_{(k)}+{ }^{I} \Gamma_{(k)}^{-1}\right)^{-1}\left(B_{(k)}^{T} M_{(k)} A_{(k)}+B_{(k)}^{T} \Sigma_{k}^{-1} A_{(k)}\right), \\
\Gamma_{(k)} & =B_{(k)}^{T} M_{(k)} B_{(k)}+B_{(k)}^{T} \Sigma_{k}^{-1} B_{(k)}+{ }^{I} \Gamma_{(k)}^{-1},
\end{aligned}
$$

where $M_{(k)}$ is the matrix of the obtained quadratic cost function from the $k$ th model, and $\Sigma_{k}$ is the covariance matrix which considers the spread around the $k$ th subpart of the state and takes the rest of the state as external variables.

II. Form 2 of the optimal controller:

The second form can be obtained by partitioning, the state, $A_{(k)}$, control $B_{(k)}$, and covariance $\Sigma_{k}$ matrices in the following forms,

$$
A_{(k)}=\left[\begin{array}{cc}
A_{k ; k} & A_{k ;-k} \\
A_{-k ; k} & A_{-k ;-k}
\end{array}\right] \quad B_{(k)}=\left[\begin{array}{c}
B_{k} \\
B_{-k}
\end{array}\right] \quad \Sigma_{k}=\left[\begin{array}{cc}
\Sigma_{k ; k} & \Sigma_{k ;-k} \\
\Sigma_{-k ; k} & \Sigma_{-k ;-k}
\end{array}\right],
$$

where according to the assumed models, $A_{k ; k}$ is a square matrix which relates the controlled states to themselves, $A_{k ;-k}$ is the matrix which relates the controlled states to the external variables, $A_{-k ; k}=0$, and $A_{-k ;-k}$ is a square matrix which relates the external variables to themselves. Here also $B_{k}$ is the control matrix of the controlled states, $B_{-k}=0$ is the control matrix of the external variables, $\Sigma_{k ; k}$ is the covariance matrix which specifies the spread on the controlled state variables, and $\Sigma_{k ;-k}=0, \Sigma_{-k ; k}=0, \Sigma_{-k ;-k}=0$.

Partition $M_{(k)}$ conformably to the partitioning of $A_{(k)}: M_{(k)}=\left[\begin{array}{cc}M_{k ; k} & M_{k ;-k} \\ M_{-k ; k} & M_{-k ;-k}\end{array}\right]$, then equation (32) is equivalent to the three equations:

a) The Riccati equation of the controlled states without the external variables,

$$
\begin{aligned}
M_{k ; k} & =A_{k ; k}^{T} \Sigma_{k ; k}^{-1} A_{k ; k}+A_{k ; k}^{T} M_{k ; k} A_{k ; k}-\left(A_{k ; k}^{T} M_{k ; k} B_{k}+A_{k ; k}^{T} \Sigma_{k ; k}^{-1} B_{k}\right) \\
& \times\left[B_{k}^{T} M_{k ; k} B_{k}+B_{k}^{T} \Sigma_{k ; k}^{-1} B_{k}+{ }^{I} \Gamma^{-1}\right]^{-1}\left(B_{k}^{T} M_{k ; k} A_{k ; k}+B_{k}^{T} \Sigma_{k ; k}^{-1} A_{k ; k}\right) .
\end{aligned}
$$

b) The equation respecting the influence of the external variables on the controlled state,

$$
\begin{aligned}
M_{k ;-k} & =A_{k ; k}^{T} \Sigma_{k ; k}^{-1} A_{k ;-k}+A_{k ; k}^{T} M_{k ; k} A_{k ;-k}+A_{k ; k}^{T} M_{k ;-k} A_{-k ;-k}-\left(A_{k ; k}^{T} M_{k ; k} B_{k}+A_{k ; k}^{T} \Sigma_{k ; k}^{-1} B_{k}\right) \\
& \times\left[B_{k}^{T} M_{k ; k} B_{k}+B_{k}^{T} \Sigma_{k ; k}^{-1} B_{k}+{ }^{I} \Gamma^{-1}\right]^{-1} \\
& \times\left(B_{k}^{T} M_{k ; k} A_{k ;-k}+B_{k}^{T} M_{k ;-k} A_{-k ;-k}+B_{k}^{T} \Sigma_{k ; k}^{-1} A_{k ;-k}\right) .
\end{aligned}
$$

c) The equation concerning external variables only,

$$
\begin{aligned}
M_{-k ;-k} & =A_{k ;-k}^{T} x \Sigma_{k ; k}^{-1} A_{k ;-k}+A_{k ;-k}^{T} M_{k ; k} A_{k ;-k}+2 A_{-k ;-k}^{T} M_{k ;-k} A_{k ;-k}+A_{-k ;-k}^{T} M_{-k ;-k} A_{-k ;-k} \\
& -\left(A_{k ;-k}^{T} M_{k ; k} B_{k}+A_{-k ;-k}^{T} M_{k ;-k} B_{k}+A_{k ;-k}^{T} \Sigma_{k ; k}^{-1} B_{k}\right)\left[B_{k}^{T} M_{k ; k} B_{k}+B_{k}^{T} \Sigma_{k ; k}^{-1} B_{k}+{ }^{I} \Gamma^{-1}\right]^{-1} \\
& \times\left(B_{k}^{T} M_{k ; k} A_{k ;-k}+B_{k}^{T} M_{k ;-k} A_{-k ;-k}+B_{k}^{T} \Sigma_{k ; k}^{-1} A_{k ;-k}\right) .
\end{aligned}
$$

With the partitioned form of the Riccati matrix, the feedback control matrix (31) is 
equivalent to,

$$
\begin{aligned}
& C_{(k)}=-\left(B_{k}^{T} M_{k ; k} B_{k}+B_{k}^{T} \Sigma_{k ; k}^{-1} B_{k}+{ }^{I} \Gamma_{(k)}^{-1}\right)^{-1} \\
& \times \quad\left(\left[B_{k}^{T} M_{k ; k} A_{k ; k} \quad B_{k}^{T} M_{k ; k} A_{k ;-k}+B_{k}^{T} M_{k ;-k} A_{-k ;-k}\right]\right. \\
& \left.+\left[B_{k}^{T} \Sigma_{k ; k}^{-1} A_{k ; k} \quad B_{k}^{T} \Sigma_{k ; k}^{-1} A_{k ;-k}\right]\right),
\end{aligned}
$$

Proof: The first part of the theorem can be proven by adapting the proof of theorem 1 in Herzallah (2012). The second part of the theorem can be proven by considering the partitioned form of the state, control, covariance and Riccati matrix as stated above.

Remark 3: The second form of the optimal control law as given by equation (36) implies that only the first two equations (33) and (34) equivalent to the Riccati need to be solved. Moreover, equation (34) determining $M_{k ;-k}$ is linear. The third equation (35), the block of the Riccati equation that relates external variables to themselves, does not need to be solved. This structure significantly decreases the computational efforts in obtaining the optimal control law within the FPD distributed control framework and also as compared to the global FPD control method.

Remark 4: Only the parts of the state matrix that relate the controlled states to themselves and to external variables, i.e. $A_{k ; k}$ and $A_{k ;-k}$ have to be estimated by the $k$ th node. Bayesian methodology Peterka (1981) serves well to this purpose and it essentially boils down to recursive least squares applied to linear auto-regression on $x_{t ; k}$ combined with regression on $x_{t ;-k}$, typically with a suitable forgetting factor in the estimation process. Either the classical version of the Bayesian methodology Kulhavý \& Zarrop (1993) or the advanced partial version Dedecius et al. (2012) can be implemented. The estimation of the parameters $A_{k ; k}$ is feasible as the $k$ th node considers the part of state having a small dimension. The dimension of $x_{t ;-k}$ and thus $A_{k ;-k}$ could generally be large when considering the full state of the network. However, according to our hypothesis it is sufficient to include only partial states of the node neighbours from $x_{t ;-k}$. Following this, the joint estimation of $\left[A_{k ; k}, A_{k ;-k}\right]$ becomes an undemanding routine task. Moreover, $A_{-k ;-k}$ can be obtained by message passing from neighbours. They are essentially state transition matrices of the closed loops of the neighbours' dynamics. This latter statement is elaborated in the next section.

\section{Algorithm of the FPD Distributed controller}

The distributed optimized controllers discussed in Section 4.2 will exchange information or messages about the parameters of the dynamics of the subparts of the system and will update these parameters iteratively in a probabilistic framework until they converge. Specifically, the $k$ th optimized randomized controller offers its neighbour the part of the resulting optimal closed loop related to the variables it perceives as external variables. It improves their description. The following algorithm can be readily applied to the neighbour distributed controllers and models and can be used for updating the parameters of the dynamics of the various subparts in the system.

- Given the initial state values $x_{t}$

- For the $k$ th node, estimate the parameters modeling the $k$ th subpart of the system states, $A_{k ; k}, A_{k ;-k}$ and $B_{k}$.

- For the $k$ th node, initialize the estimate of parameters describing the $-k$ th subpart of the system states, $A_{-k ;-k}$.

- At each time instant $t$

- Loop

- Use equation (33) to calculate the part of the Riccati matrix, $M_{k ; k}$ determining the feedback gain of the $k$ th controller from controlled states.

- Use the linear equation (34) to calculate the part of the Riccati matrix, $M_{k ;-k}$ deter- 
mining the feedback gain of the $k$ th controller from "external" variables.

- Use equation (36) to calculate the feedback control matrix $C_{(k)}$.

- Update the parameters for "external" variables of the $j$ th subpart of the system as follows,

$$
x_{t+1 ;(j) \leftarrow(k)} \Rightarrow A_{(j) ;-j} x_{t}=\left(A_{(k) ; k}+B_{(k) ; k} C_{(k)}\right) x_{t}
$$

where $x_{t+1 ;(j) \leftarrow(k)}$ is used to denote models of the $j$ th node as obtained from the $k$ th node. The optimally tuned closed loop from the view point of the $k$ th controller has the form given in equation (29), repeated here,

$$
x_{t+1 ;(k)}=\left(A_{(k)}+B_{(k)} C_{(k)}\right) x_{t}+B_{(k)} \epsilon_{t ;(k)}+\omega_{t+1 ;(k)} .
$$

To further explain Equation (37), we implement it on a system consisting of two states. The system is first decomposed into two subsystems or nodes, where the first node is controlled by a control signal $u_{t,(1)}$ and the second node is controlled by a control signal $u_{t,(2)}$. The first node is concerned with the regulation of the first state, hence takes the second state as an external signal. Consequently, this node is governed by the following model,

$$
x_{t+1,(1)}=\left[\begin{array}{c}
x_{t+1,(1 ; 1)} \\
x_{t+1,(1 ; 2)}
\end{array}\right]=\left[\begin{array}{cc}
a_{1 ; 1} & a_{1 ;-1} \\
0 & a_{-1 ;-1}
\end{array}\right]\left[\begin{array}{c}
x_{t, 1} \\
x_{t, 2}
\end{array}\right]+\left[\begin{array}{c}
b_{1} \\
0
\end{array}\right] u_{t,(1)}
$$

The second node on the other hand focuses on the regulation of the second state and takes the first state as an external signal, hence governed by the following model,

$$
x_{t+1,(2)}=\left[\begin{array}{c}
x_{t+1,(2 ; 1)} \\
x_{t+1,(2 ; 2)}
\end{array}\right]=\left[\begin{array}{cc}
a_{-2 ;-2} & 0 \\
a_{2 ;-2} & a_{2 ; 2}
\end{array}\right]\left[\begin{array}{c}
x_{t, 1} \\
x_{t, 2}
\end{array}\right]+\left[\begin{array}{c}
0 \\
b_{2}
\end{array}\right] u_{t,(2)}
$$

According to Equation (38), the optimally tuned closed loop from the view point of the 1st controller, neglecting the noise terms to simplify the presentation, is given by,

$$
x_{t+1,(1)}=\left[\begin{array}{l}
x_{t+1,(1 ; 1)} \\
x_{t+1,(1 ; 2)}
\end{array}\right]=\left[\begin{array}{cc}
a_{1 ; 1} & a_{1 ;-1} \\
0 & a_{-1 ;-1}
\end{array}\right]\left[\begin{array}{l}
x_{t, 1} \\
x_{t, 2}
\end{array}\right]+\left[\begin{array}{c}
b_{1} \\
0
\end{array}\right]\left[\begin{array}{cc}
c_{1 ; 1} & c_{1 ; 2}
\end{array}\right]\left[\begin{array}{l}
x_{t, 1} \\
x_{t, 2}
\end{array}\right]
$$

and the optimally tuned closed loop from the view point of the 2nd controller, neglecting the noise terms to simplify the presentation, is given by,

$$
x_{t+1,(2)}=\left[\begin{array}{c}
x_{t+1,(2 ; 1)} \\
x_{t+1,(2 ; 2)}
\end{array}\right]=\left[\begin{array}{cc}
a_{-2 ;-2} & 0 \\
a_{2 ;-2} & a_{2 ; 2}
\end{array}\right]\left[\begin{array}{l}
x_{t, 1} \\
x_{t, 2}
\end{array}\right]+\left[\begin{array}{c}
0 \\
b_{2}
\end{array}\right]\left[\begin{array}{cc}
c_{2 ; 1} & c_{2 ; 2}
\end{array}\right]\left[\begin{array}{l}
x_{t, 1} \\
x_{t, 2}
\end{array}\right]
$$

By comparing Equations (41) and (42), the two optimized controllers will exchange information about the parameters of the dynamics of the two nodes and will then update these parameters according to the following equations,

$$
\begin{aligned}
a_{-2 ;-2} & =a_{1 ; 1}+b_{1} c_{1 ; 1} \\
0 & =a_{1 ;-1}+b_{1} c_{1 ; 2} \\
0 & =a_{2 ;-2}+b_{2} c_{2 ; 1} \\
a_{-1 ;-1} & =a_{2 ; 2}+b_{2} c_{2 ; 2}
\end{aligned}
$$




\section{Coupled Map Lattice Network and Numerical Results}

We validate and illustrate the distributed probabilistic control method presented in Section 4.2 using the stochastic version Herzallah (2012) of a coupled map lattice (CML) with a periodic boundary condition as an example of complex dynamical networks, which was originally introduced in Gang \& Zhilin (1994). The obtained results of the distributed probabilistic controllers will also be compared to the global pinning control solution to the considered CML problem as discussed in section 4.1. We first give an overview of the coupled map lattice control problem.

\subsection{Overview of Coupled Map Lattice}

Consider a CML consisting of $L$ nodes with periodic boundary conditions,

$$
z_{t+1}^{j}=F\left(z_{t}^{j-1}, z_{t}^{j}, z_{t}^{j+1}\right)=f\left[(1-2 \varepsilon) z_{t}^{j}+\varepsilon\left(z_{t}^{j-1}+z_{t}^{j+1}\right)\right]+\kappa_{t+1}^{j},
$$

where $j=1,2, \ldots, L$ are the lattice sites, $L$ is the system size, $\varepsilon$ is the coupling strength, $\kappa_{t+1}^{j}$ is an additive noise signal assumed to have zero mean Gaussian distribution of covariance $\rho$, and the periodic boundary conditions are given by $z_{t}^{j+L}=z_{t}^{j}$. The local map $f(z)$ is defined to be a nonlinear function of the following form

$$
f(z)=a z(1-z)
$$

This coupled map lattice exhibits chaotic characteristics in the regime $3.57<a \leq 4.0$ and has a homogeneous steady state $z^{\star}=1-1 / a$.

The control objective here is to stabilise the homogeneous state of the lattice. Because of the complexity and the large number of nodes of the CML, this goal is conventionally achieved via pinning control methodology by applying $M$ periodically control actions placed at sites $\left\{j_{1}, \ldots, j_{M}\right\}$ in the following way

$$
z_{t+1}^{j}=F\left(z_{t}^{j-1}, z_{t}^{j}, z_{t}^{j+1}\right)+\sum_{m=1}^{M} \delta\left(j-j_{m}\right) u_{t}^{m}+\kappa_{t+1}^{j},
$$

where $\delta(0)=1$ and zero otherwise, $u_{t}^{m}$ is the control action at time $t$ applied at site $m$.

Traditionally in pinning control the theory of linear quadratic control is used to calculate the feedback control actions. For that purpose Equation (43) is linearized about the homogeneous steady state $\mathbf{z}_{t}=\left(z_{1}^{\star}, \ldots, z_{L}^{\star}\right)$ to become as follows

$$
x_{t+1}=A x_{t}+B u_{t}+\omega_{t+1},
$$

in which $x=\mathbf{z}-\mathbf{z}^{\star}$ represents the state vector, the $L \times L$ Jacobian matrix $A$, is given by

$$
A=\alpha\left[\begin{array}{ccccc}
1-2 \varepsilon & \varepsilon & 0 & \ldots & \varepsilon \\
\varepsilon & 1-2 \varepsilon & \varepsilon & \ldots & 0 \\
0 & \varepsilon & 1-2 \varepsilon & \ldots & 0 \\
\vdots & \vdots & \vdots & \ddots & \vdots \\
\varepsilon & 0 & 0 & \ldots & 1-2 \varepsilon
\end{array}\right]
$$

where $\alpha=\left.\frac{\partial f(z)}{\partial z}\right|_{z=z^{\star}}$, and $B$ is an $L \times M$ control matrix with $B_{j i}=\sum_{m} \delta(i-m) \delta\left(j-j_{m}\right)$. 
Following the discussion in Section 4.1 the pinning controlled network as a result of the designed randomised controller (21) will have the form given by equation (20), repeated here

$$
x_{t+1}=(A+B C) x_{t}+B \epsilon_{t}+\omega_{t+1},
$$

where $C$ is calculated as specified by equation (22). In Grigoriev et al. (1997), the control matrix, $B$ is chosen such that the network of systems is made controllable as opposed to stabilisable. Since the coupled map lattice considered has parity symmetry Grigoriev et al. (1997), the eigenvalues of its Jacobian are doubly degenerate. Therefore, the minimal number of controllers that yields a controllable system in this case is two, which will be assumed in this paper.

In contrast to the pinning control method, the proposed distributed control method considers designing and optimizing $K$ number of controllers, each is responsible for controlling and regulating a predefined collective number of neighbouring nodes in the lattice. For the linearized version of CML (46), the distributed controllers can be designed as discussed in Section 4.2. In the following numerical simulations, for both pinning and distributed control, the conditional means of the controllers, represented by $C x_{t}$ and $C_{(k)} x_{t}$ respectively, will be taken as inputs to the system. Here $C$ is to be calculated as specified by equation (22), and $C_{(k)}$ as specified by equation (36).

\subsection{Numerical Results of Global FPD}

The example considered here is for the logistic coupled map lattice, $f(z)=a z(1-z)$ in its nonchaotic regime with $a=3.0, \varepsilon=0.33$ and $L=5$ and with an external Gaussian random input, $\omega_{t+1}$ affecting the dynamics. Following the pinning control method Herzallah (2012), two controllers are used and they are placed next to each other at the sides of the lattice. Hence the equation of the coupled map lattice becomes:

$$
x_{t+1}=A x_{t}+B u_{t}+\omega_{t+1} \text {, }
$$

where

$$
\begin{gathered}
A=\left[\begin{array}{ccccc}
-0.34 & -0.33 & 0 & 0 & -0.33 \\
-0.33 & -0.34 & -0.33 & 0 & 0 \\
0 & -0.33 & -0.34 & -0.33 & 0 \\
0 & 0 & -0.33 & -0.34 & -0.33 \\
-0.33 & 0 & 0 & -0.33 & -0.34
\end{array}\right], \\
B=\left[\begin{array}{ll}
1 & 0 \\
0 & 0 \\
0 & 0 \\
0 & 0 \\
0 & 1
\end{array}\right] \quad \Sigma=E\left[\omega_{t+1} \omega_{t+1}^{T}\right]=0.001 I_{5 \times 5} .
\end{gathered}
$$

The lattice is initially at time $t=0$ in state $x=0.25$ and the aim is to return the lattice to the origin (the fixed point position) or a state close to the origin. The matrices $A$ and $B$ and the covariance matrix $\Sigma>0$ are assumed to be known. The covariance matrix of the controller is taken to be ${ }^{I} \Gamma=0.1 I_{2 \times 2}$. The resulted states of the lattice network and the obtained control efforts are illustrated in Figures 2(a) and (b), respectively, which show that the controlled network is globally synchronized by the designed global probabilistic pinning controller. Please note that the fluctuations of the state values around the origin is due to the stochastic nature of the considered variant of coupled map lattice. If the additive noise term $\omega_{t+1}$ was to be neglected, all states of the coupled map lattice would have attained an exact value of zero. Figure 2(c) gives the solution of 
the control matrix $C$. This figure shows that the control effort is larger for those sites that are far away from the pinning site. This is expected as reported in Herzallah (2012) and means that the perturbation introduced by the controllers decays with the increasing distance to the pinning site. These obtained results will be compared to the distributed control results which will be discussed in the following section.

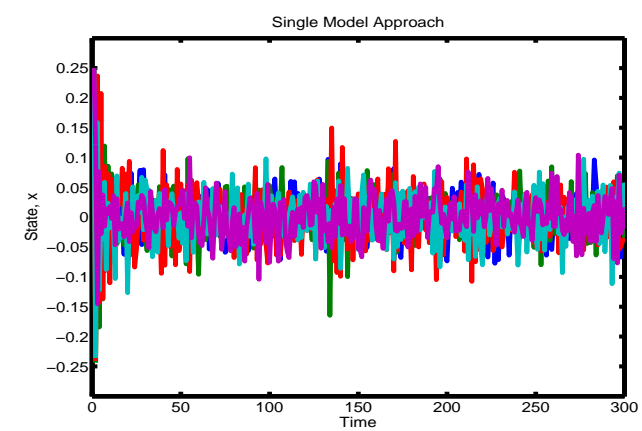

(a)

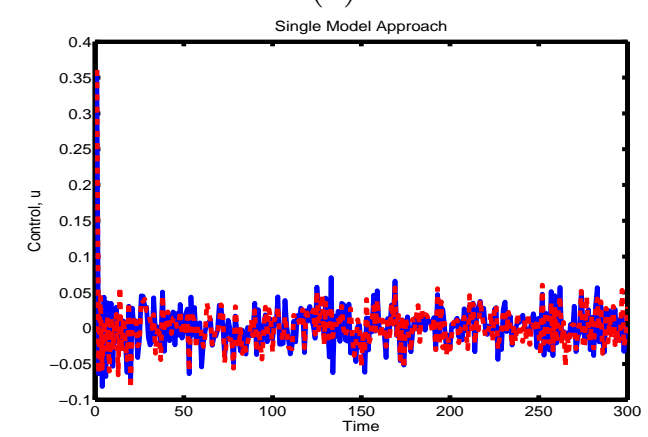

(b)

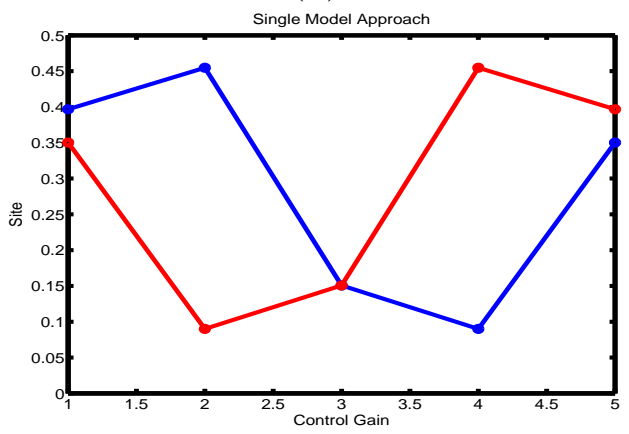

(c)

Figure 2. States and control efforts of a non chaotic coupled map lattice with, $L=5, a=3$, and $\varepsilon=0.33$ as a result of the global FPD controller, Section 4.1, Equation (22): (a) states as a result of the global FPD controller. (b) control efforts from the global controller. (c) control gain of the global controller.

\subsection{Numerical Results of Distributed FPD Control}

For comparison purposes, the same logistic coupled map lattice, $f(z)=a z(1-z)$ in its non-chaotic regime with $a=3.0, \varepsilon=0.33$ and $L=5$ and with an external Gaussian random input, $\omega_{t+1}$ affecting the dynamics will be considered here for the design of distributed probabilistic controller. We design two distributed probabilistic controllers $u_{t ;(1)}$ and $u_{t ;(2)}$. The first probabilistic controller $u_{t ;(1)}$ is concerned with the regulation of the first three nodes in the lattice while modelling the 
rest of the states as an external observable disturbance,

$$
x_{t+1 ;(1)}=A_{(1)} x_{t}+B_{(1)} u_{t ;(1)}+\omega_{t+1 ;(1)},
$$

where

$$
\begin{aligned}
A_{(1)}= & {\left[\begin{array}{ccccc}
-0.34 & -0.33 & 0 & 0 & -0.33 \\
-0.33 & -0.34 & -0.33 & 0 & 0 \\
0 & -0.33 & -0.34 & -0.33 & 0 \\
0 & 0 & 0 & a_{(1) ; 44} & 0 \\
0 & 0 & 0 & 0 & a_{(1) ; 55}
\end{array}\right], \quad B_{(1)}=\left[\begin{array}{l}
1 \\
1 \\
1 \\
0 \\
0
\end{array}\right] } \\
& \text { and } \Sigma_{1}=E\left[\omega_{t+1 ;(1)} \omega_{t+1 ;(1)}^{T}\right]=\left[\begin{array}{cc}
0.001 I_{3 \times 3} & 0_{3 \times 2} \\
0_{2 \times 3} & 0_{2 \times 2}
\end{array}\right],
\end{aligned}
$$

because the last two states are taken as external variables. The second probabilistic controller $u_{t ;(2)}$ is concerned with the regulation of the last three nodes in the lattice while modelling the rest of the states as an external observable disturbance,

$$
x_{t+1 ;(2)}=A_{(2)} x_{t}+B_{(2)} u_{t ;(2)}+\omega_{t+1 ;(2)},
$$

where

$$
\begin{gathered}
A_{(2)}=\left[\begin{array}{ccccc}
a_{(2) ; 11} & 0 & 0 & 0 & 0 \\
0 & a_{(2) ; 22} & 0 & 0 & 0 \\
0 & -0.33 & -0.34 & -0.33 & 0 \\
0 & 0 & -0.33 & -0.34 & -0.33 \\
-0.33 & 0 & 0 & -0.33 & -0.34
\end{array}\right], \quad B_{(2)}=\left[\begin{array}{l}
0 \\
0 \\
1 \\
1 \\
1
\end{array}\right] \\
\Sigma_{2}=E\left[\omega_{t+1 ;(2)} \omega_{t+1 ;(2)}^{T}\right]=\left[\begin{array}{cc}
0_{2 \times 2} & 0_{2 \times 3} \\
0_{3 \times 2} & 0.001 I_{3 \times 3}
\end{array}\right] .
\end{gathered}
$$

because, the first two states are taken as external variables. The four unknown parameters $a_{(1) ; 44}, a_{(1) ; 55}, a_{(2) ; 11}$, and $a_{(2) ; 22}$ are related to the external observable states from the point of view of the first and second controllers and are initialized randomly. The two controllers then exchange information about the closed loop response of the system which is used to update these unknown parameters as discussed in Section 4.2.

Similarly here for comparison purpose, the lattice is assumed to be initially at time $t=0$ in state $x=0.25$ and the aim is to return the lattice to the origin (the fixed point position) or a state close to the origin. The covariance matrices of the first and second controllers are taken to be ${ }^{I} \Gamma_{(1)}=0.1,{ }^{I} \Gamma_{(2)}=0.1$ respectively. The resulted states of the lattice network and the obtained control efforts are illustrated in Figures 3(a) and (b), respectively. As can be seen from these figures, although the distributed probabilistic controllers are optimised independently using local information of the controlled subsystems, they are very effective in globally regulating the controlled CML network. Obtained control results are very close to the global probabilistic pinning control solution. Similarly here, the fluctuations of the state values around the origin is due to the stochastic nature of the considered variant of coupled map lattice. Figure 3(c) gives the solution of the control matrices $C_{1}$ and $C_{2}$. This figure, as expected, shows that the control effort is larger for those sites controlled by the corresponding controller and smaller for those sites whose states are perceived as external variables. 


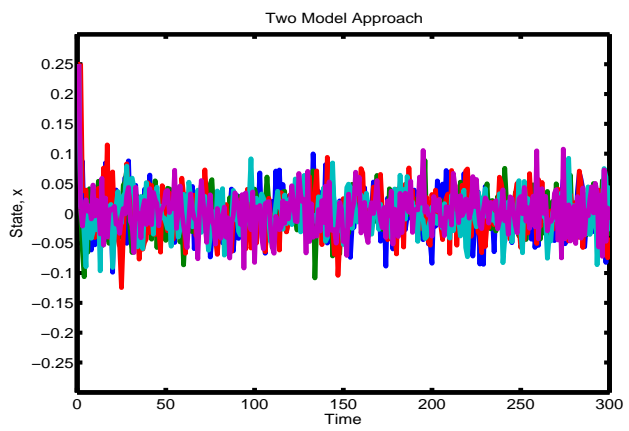

(a)

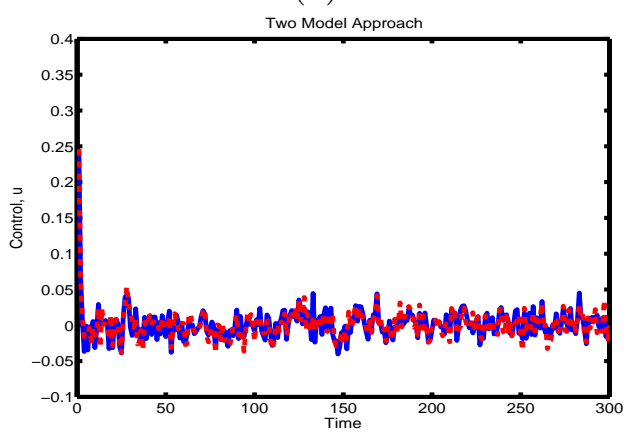

(b)

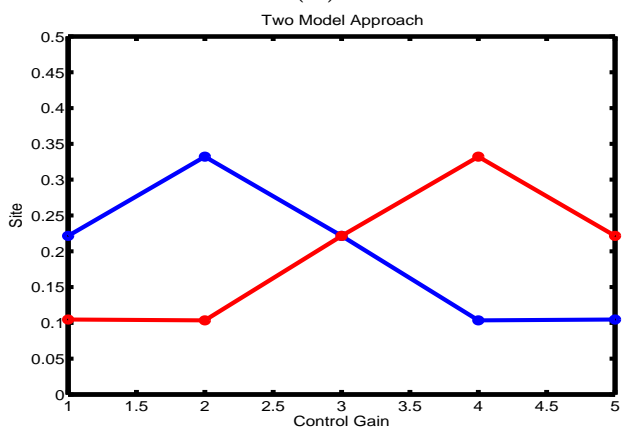

(c)

Figure 3. States and control efforts of a non chaotic coupled map lattice with, $L=10, a=3$, and $\varepsilon=0.33$ as a result of the distributed FPD controller, Section 4.2, Equation (36): (a) states as a result of the distributed FPD controller. (b) control efforts from the distributed controller. (c) control gain of the distributed controllers.

\section{Conclusion}

The problem of controlling complex dynamical systems with large number of nodes has been considered in this paper. The proposed solution is developed via distributed stochastic control where noise and uncertainties are contained using probabilistic approaches. This solution considers a bottom up strategy where simple controllers are designed to focus on the control of the individual subparts of the system either completely independently, or within various structures like cascade control. By utilising the FPD approach, we developed the general methodology for distributed control proposed in this paper. The developed distributed controllers exchange information about the various nodes in the network and use this information to update their governing dynamical relations. The theoretical findings were then validated on a coupled map lattice network as an example of complex dynamical networks. Numerical results confirm the effectiveness of the proposed distributed probabilistic control approach in globally regulating the states of the network. This makes the outlined research direction worth developing. Surely, it is necessary and possible to 
elaborate on

- a direct analogy to controlled Markov chains (Markov Decision Processes);

- adaptive versions of local controllers;

- sharing of model parts when they result from estimation and thus they are uncertain, cf. Kárný et al. (2009); Sečkárová (2013).

It is desirable but hard to

- generalise the analogy of the second form of the optimal controller, which is the main tool for decreasing the overall complexity, to FPD;

- analyse emerging properties (stability, stationary behaviour, control quality) of the proposed distributed control;

- apply the proposed control on non-trivial practical problems.

Acknowledgment: The research was partially supported by GAČR 13-13502S.

\section{References}

Aji, S. M., \& McEliece, R. J. (2000). The generalized distributed law. IEEE Transactions on Information Theory, 46 (2),pp. 325-343.

Boccaletti, S. , Kurths, J. , Osipov, G. , Valladares, D. L., \& Zhou, C. S. (2002). The synchronization of chaotic systems. Physics Reports, 366,pp. 1-101.

Gallager, R. G. (1963). Low-Density Parity Check Codes. M.I.T. Press, Cambridge, MA.

Gang, H., \& Zhilin, Q. (1994). Controlling spatiotemporal chaos in coupled map lattice systems. Physical Review Letters, 72(1), pp. 68-73.

Grigoriev, R. O., Cross, M. C., \& Schuster, H. G. (1997). Pinning control of spatiotemporal chaos. Physical Review Letters, 79 (15), pp. 2795-2798.

Guy, T. V., \& Kárný, M. (2005). Stationary fully probabilistic control design. In J. Filipe, J. A. Cetto, and J. L. Ferrier (Eds.), Proc. of the Second Int. Conference on Informatics in Control, Automation and Robotics, Barcelona, 2005. INSTICC (pp. 109-112).

Herzallah, R. \& Kárný, M. (2011). Fully probabilistic control design in an adaptive critic framework. Neural Networks, 24 (4), pp. 1128-1135.

Kulhavý, R. \& Zarrop, M. B. (1993). On a general concept of forgetting. Int. J. of Control, 58(4), pp. 1128-1135.

Dedecius, K., Nagy, I. \& Kárný M. (2012). Parameter tracking with partial forgetting method. Int. J. of Adaptive Control and Signal Processing, 26(1),pp. 1-12.

Herzallah, R. (2012). Probabilistic synchronisation of pinning control. International Journal of Control, pp. 1-9. DOI:10.1080/00207179.2012.700488.

Herzallah, R. (2011). Enhancing the performance of intelligent control systems in the face of higher levels of complexity and uncertainty. International Journal of Modelling Identification and Control, 12(4), pp. $311-327$.

Herzallah, R. \& Lowe D. (2003). Robust control of nonlinear stochastic systems by modeling conditional distributions of control signals. Neural Computing and Applications, 12, pp. 98-108.

Herzallah, R. \& Lowe D. (2007). Distribution modelling of nonlinear inverse controller under a Bayesian framework. IEEE Transactions on Neural Networks, 18(1), pp. 107-114.

Herzallah, R. (2013). Probabilistic DHP adaptive critic for nonlinear stochastic control systems. Neural Networks, 42, pp. 74-82.

Kárńy, M. (1996). Towards fully probabilistic control design. Automatica, 32(12), pp. 1719-1722.

Kárný, M., Böhm, J., Guy, T. V., Jirsa, L., Nagy, I., Nedoma, P. \& Tesař, L. (2006). Optimized Bayesian Dynamic Advising: Theory and Algorithms. Springer, 2006.

Kárný, M., \& Guy, T. V. (2006). Fully probabilistic control design. Systems 83 Control Letters, 55 (4), pp. $259-265$.

Kárný, M., Guy, T. V., Bodini, A., \& Ruggeri, F. (2009). Cooperation via sharing of probabilistic information. 
Int. J. of Computational Intelligence Studies, pp. 139-162.

Meditch, J. S. (1969). Stochastic Optimal Linear Estimation and Control. Mc. Graw Hill, 1969.

Parisi, G., Mézard, M., \& Virasoro, M. A. (1987). Spin Glass Theory and Beyond. World Scientific, Singapore, 1987.

Pearl, J. (1988). Probabilistic Reasoning in Intelligent Systems. Morgan Kaufman, San Mateo, CA, 1988.

Peterka, V. (1981). Bayesian system identification. In P. Eykhoff, editor, Trends and Progress in System Identification, pp. 239-304. Pergamon Press, Oxford, 1981.

Porfiri, M., \& diBernardo, M. (2008). Criteria for global pinning controllability of complex networks. Automatica, 44, pp. 3100-3106.

Rao, C.R. (1987). Linear method of statistical inference and their applications. Academia, Prague, 1987. in Czech.

Sečkárová, V. (2013). On supra-Bayesian weighted combination of available data determined by Kerridge inaccuracy and entropy. Pliska Stud. Math. Bulgar., 44, pp. 159-168.

Šiljak, D. D., \& Zečević, A. I. (2005). Control of large scale systems: Beyond decentralised feedback. Annual Reviews in Control, 29, pp. 169-179.

Van den Broek, B., Wiegerinck, W., \& kappen, B. (2008). Graphical model inference in optimal control of stochastic multi-agent systems. Journal of Artifial Intelligence Research, 33, pp. 95-122.

Wang, Z., Lu, R., \& Shen, B. (2014). Distributed estimation and control for general systems. International Journal of General Systems, 43(3-4), pp. 247-253.

Xiaohui Tan, Jiye Zhang, \& Yiren. Yang. (2003). Synchronizing chaotic systems using backstepping design. Chaos, Solitons and Fractals, 16, pp. 37-45.

Zdeborová, L., Decelle, A., \& Chertkov, M. (2009). Message passing for optimization and control of a power grid: Model of a distribution system with redundancy. Phys. Rev. E, 80, 046112. 